\title{
Diacronie
}

Studi di Storia Contemporanea

$N^{\circ} 35,3 \mid 2018$

Gli strumenti di Clio

\section{Commento di Eugenio Guccione a Un'età contro la storia. Saggio sulla rivoluzione del XXI secolo}

\section{Eugenio Guccione}

\section{(2) OpenEdition}

Journals

\section{Edizione digitale}

URL: http://journals.openedition.org/diacronie/9103

DOI: 10.4000/diacronie. 9103

ISSN: 2038-0925

\section{Editore}

Association culturelle Diacronie

\section{Notizia bibliografica digitale}

Eugenio Guccione, "Commento di Eugenio Guccione a Un'età contro la storia. Saggio sulla rivoluzione del XXI secolo », Diacronie [Online], № 35, 3 | 2018, documento 11, Messo online il 29 septembre 2018, consultato il 01 mai 2019. URL : http://journals.openedition.org/diacronie/9103; DOI : 10.4000/ diacronie.9103 


\section{Diacronie}

Studi di Storia Contemporanea

\section{$35,3 / 2018$}

Gli strumenti di Clio: uomini, luoghi e teorie della storia dalla tradizione critica alla comunicazione digitale

\section{Commento di Eugenio Guccione a Un'età contro la storia. Saggio sulla rivoluzione del XXI secolo}

\section{Eugenio GUCCIONE}

Per citare questo articolo:

GUCCIONE, Eugenio, «Commento di Eugenio Guccione a Un 'età contro la storia. Saggio sulla rivoluzione del XXI secolo», Diacronie. Studi di Storia Contemporanea : Gli strumenti di Clio: uomini, luoghi e teorie della storia dalla tradizione critica alla comunicazione digitale, 35, 3/2018, 29/09/2018,

URL: < http://www.studistorici.com/2018/09/29/guccione_numero_35/ >

Diacronie Studi di Storia Contemporanea $\rightarrow$ http://www.diacronie.it

Rivista storica online. Uscita trimestrale.

redazione.diacronie@hotmail.it

Comitato di direzione: Naor Ben-Yehoyada - João Fábio Bertonha - Christopher Denis-Delacour - Maximiliano Fuentes Codera Anders Granås Kjøstvedt - John Paul Newman - Deborah Paci - Niccolò Pianciola - Spyridon Ploumidis - Wilko Graf Von Hardenberg Comitato di redazione: Jacopo Bassi - Luca Bufarale - Gianluca Canè - Fausto Pietrancosta - Alessandro Salvador - Matteo Tomasoni Diritti: gli articoli di Diacronie. Studi di Storia Contemporanea sono pubblicati sotto licenza Creative Commons 3.0. Possono essere

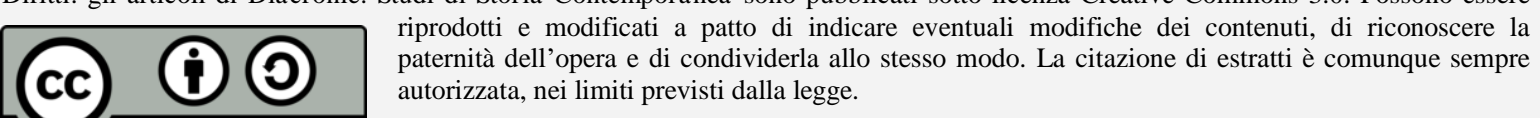




\title{
11/ Commento di Eugenio Guccione a Un'età contro la storia. Saggio sulla rivoluzione del XXI secolo
}

\author{
Eugenio GUCCIONE
}

Eugenio Guccione nel suo commento al saggio di Giuseppe Carlo Marino su globalizzazione e crisi del "pensare storico" propone una lettura "storico-filosofica" delle mutazioni avvenute nel rapporto tra avvenimenti politici, ruolo e significato dell 'esistenza umana, e dibattito culturale legato ai fenomeni "globalizzanti", inserendo gli eventi e le evoluzioni recenti del pensiero storico in un contesto più ampio di ridefinizione del concetto stesso di storia.

A quanti tra i lettori hanno una visione metafisica e non immanentistica della realtà, ivi compresa la storia degli uomini, sarà difficile o quasi impossibile condividere in toto la pur affascinante riflessione di Giuseppe Carlo Marino, riportata nel suo recente lavoro, Un'età contro la storia - Saggio sulla rivoluzione del XXI secolo ${ }^{1}$. Io, per formazione e per consapevole scelta, mi ritrovo tra quelli. Tuttavia, nonostante la mia estrema divergenza dalle premesse di carattere filosofico, sono d'accordo qua e là su molte intuizioni dell'autore, che, costruendosi una base di partenza e «sfruttando le risorse» che gli pervengono, com'egli precisa, «da una mai interrotta fedeltà alla lezione di Antonio Gramsci», tende a rilevare che «la rivoluzione del XXI secolo sta delineando gli sviluppi di "un'età contro la storia"»².

A parte il costante uso di certa terminologia nel presentare come un «mito» la Genesi, primo libro della Bibbia, Marino sostiene che l'uomo nell'Eden non sia una creatura libera, bensì sia una creatura necessitata, vincolata da «leggi naturali», le quali non potevano non provenire che da Dio. Egli scrive: «In quel mitico Eden, ovvero nel cosiddetto stato di natura, niente era da considerarsi libero che non fosse necessario, vincolato a regole (quelle che si chiamano "leggi naturali") che ne fissavano appunto la necessità, prima del tempo storico e pertanto in una

\footnotetext{
${ }^{1}$ MARINO, Giuseppe Carlo, Un'età contro la storia. Saggio sulla rivoluzione del XXI secolo, Palermo, Università degli Studi di Palermo, Dipartimento di Scienze Politiche e delle relazioni internazionali (DEMS), 2017.

${ }^{2}$ Ibidem, pp. 5, 7.
} 
condizione radicalmente senza storia»" ${ }^{3}$ Ma ci sia consentito osservare che se così veramente fosse stato, l'uomo non avrebbe mai potuto disubbidire a Dio. L'«individuo necessitato» non ha facoltà d'agire con propria volontà. La sua vita, ingabbiata nella necessità, si articolerebbe in maniera deterministica.

L'uomo, invece, proprio perché libero, ossia nella condizione di scegliere il bene o il male, poté ribellarsi al Creatore. E rimarrà libero anche dopo l'uscita o la cacciata dall'Eden. Il non riconoscere libertà d'azione ad Adamo nel paradisiaco stato di natura è un preambolo che ipoteca gran parte del successivo ragionamento dell'autore. Questi è portato a distinguere un periodo «radicalmente senza storia» - quello della permanenza dell'uomo nell'Eden -, da un periodo di inizio effettivo della storia, di cui l'individuo, nel pieno esercizio della sua libertà, è il vero e l'unico artefice.

Occorrerebbe, piuttosto, tenere conto che l'esistenza umana sin dagli esordi è contaminata dalla tentazione e dall'abuso del libero arbitrio, che tende ad affrancarsi da un sempre possibile controllo divino. A tal proposito, se per storia si voglia intendere in senso polibiano la raccolta e la narrazione dei fatti ovvero in senso vichiano la loro interpretazione e comprensione come manifestazione dello spirito umano o dello stesso spirito universale, si dovrebbe, a mio avviso, parlare sempre di due tipi di storia, sostanzialmente diversi, in cui, però, il soggetto agente continua a essere lo stesso: l'uomo libero. Egli, prima, vive nell'amicizia con Dio e, dopo, in una situazione del tutto capovolta e compromessa dal peccato originale, dà inizio a un nuovo corso delle sue vicende nell'esigenza, più o meno consapevole, di recuperare i perduti vantaggi dell'Eden. Ci troviamo, secondo una espressione di papa Joseph Ratzinger, di fronte al «dramma della libertà, che Dio accetta fino in fondo per amore» (Udienza dell's dicembre 2008) ${ }^{4}$.

La tesi dell'autore, caratterizzandosi con alte punte di originalità e di innovazione in campo storiografico, non è sviluppata sulla scia delle sole teorie gramsciane. Qua e là si colgono espliciti richiami a molti altri pensatori antichi e moderni, tra i quali un posto privilegiato è riservato a Karl Marx per le condivise interpretazioni socio-economiche e storiche. Si nota, tuttavia, l'assenza di Alexis de Tocqueville, che, da acuto analista liberale, si pose il problema della congenita degenerazione della democrazia e ne indicò adeguati rimedi relativamente alle disfunzioni e alla pericolosa tendenza al socialismo. Un politologo di riferimento molto valido e convincente, che, nelle diagnosi dei regimi democratici, è tuttora in grado di offrire spunti, suggerimenti e soluzioni ${ }^{5}$.

\footnotetext{
${ }^{3}$ Ibidem, p. 12.

${ }^{4}$ Atto di venerazione all'Immacolata a Piazza di Spagna, Discorso del Santo Padre Benedetto XVI, Solennità dell'Immacolata Concezione della Beata Vergine Maria, pronunciato l'8 dicembre 2008, URL:

<http://w2.vatican.va/content/benedict-xvi/it/speeches/2008/december/documents/hf_benxvi_spe_20081208_immacolata.html> [consultato il 19 luglio 2018].

${ }^{5} \mathrm{Si}$ vedano CABOARA, Lorenzo, Democrazia e libertà del pensiero di Alexis de Tocqueville, Milano, Hoepli, 1946;
} 
Tale segnalazione, comunque, non vuole essere affatto un rilievo a Marino. Un saggio, «nelle forme proprie di un pamphlet», non può essere appesantito dalle usuali citazioni accademiche. E, in più, vada dato atto all'autore, che, quantunque egli proceda in rigorosa coerenza con le premesse, rifugge da esclusive scelte ideologiche. Tanto è vero che egli si richiama anche a pensatori di tutt'altra sponda, come Agostino, Vico, Kant, Hegel, Croce, Gentile, Maritain. E, addirittura, cita favorevolmente per ben tre volte papa Francesco, che, al timone della Chiesa cattolica, sembra a lui essere il solo a «sfuggire» alla «vocazione reazionario-totalitaria del "pensiero unico" del capitalismo globalizzato» e impegnato a condurre una «strategia sopraffattrice» di assimilazione dei poveri interni ed esterni all'Occidente ${ }^{6}$.

Marino, sottolineando che - «molto al di là di quel che di innovativo in proposito sembrava aver avviato Giovanni Paolo II» - l'attuale papa si distingua sia per le ripetute denunce dello stato di schiavitù delle masse dei migranti affluenti all'Occidente, sia per la linea di condotta del suo "grande pontificato» contrassegnato dalla «fine definitiva dell'ideologia delle Crociate» e aperto al dialogo con altri credi religiosi, sia per l'allarme lanciato all'umanità sull'avvenuta esplosione di una «nuova "guerra mondiale a pezzi" (ovvero a tappe), manifesta in forme molteplici e con dinamica sincopata».

Ciò porta lo storico "gramsciano» a esprimere un giudizio sostanzialmente positivo sulla Chiesa cattolica, la quale - egli scrive - «in un inedito slancio di progettualità democratica, solidaristica e liberatrice sta recidendo del tutto certi fili che l'avevano impigliata in innaturali complicità ed alleanze con le gerarchie sociali e con gli interessi del vecchio capitalismo». Di contro, «la mentalità neocapitalistica della globalizzazione, sta accentuando [...] una sua risorgente vocazione autoritario-totalitaria dalle indubbie ascendenze imperialistiche, per quanto venga occultata e ufficialmente condannata» ${ }^{7}$.

Se si considerano i vari specifici tipi di capitalismo e di neocapitalismo, come quelli americano, russo e cinese in costante lotta tra di loro, debbo confessare che non riesco a cogliere l'esistenza di un «unico fronte neocapitalista» con un'unica strategia. Di un paradossale «modello asiatico di capitalismo di Stato [...] - come l'autore esattamente scrive fu Stalin il demiurgo luciferino, un modello, e quindi nella sua pratica attuazione un sistema, al quale [...] ci si ostinò ad attribuire il nome del socialismo» ${ }^{8}$. Mi pare, pur con la mia incompetenza in campo economico, che ci siano diversi neocapitalismi con proprie caratteristiche, proprie forze e proprie zone di influenza. E il conflitto fra di loro è subdolo. È

MANENT, Pierre, Tocqueville et la nature de la démocratie, Paris, Gallimard, 2006; CATANORCHI, Olivia, RAGAZZONI, David (a cura di), Il destino della democrazia. Attualità di Tocqueville, Roma, Edizioni di Storia e Letteratura, 2010.

${ }^{6}$ MARINO, Giuseppe Carlo, Un'età contro la storia. Saggio sulla rivoluzione del XXI secolo, cit., pp. 87-89.

${ }^{7}$ Ibidem, pp. 89-90.

${ }^{8}$ Ibidem, p. 31. 
impossibile prevedere quale fra tutti prevarrà o se il futuro riserva loro ulteriori frammentazioni o aggregazioni.

Potrei anche essere d'accordo sulla constatazione di Marino, ma sono indotto a dedurne che se così stanno effettivamente le cose, - cioè da un lato la Chiesa la quale, nella sua visione universale, si evolve democraticamente e dall'altro il neocapitalismo il quale, nell'ambito della globalizzazione, procede con una «sua risorgente vocazione autoritario-totalitaria» - il tanto temuto pericolo dell'egemonia di un «pensiero unico» si allontana, sino a lasciare presumere un annientamento.

La specifica categoria del neocapitalismo, ritenuta «inesorabile» nel saggio e volta alla «eliminazione delle diversità e delle differenze», sarebbe destinata, in altri termini, a fare cilecca. Il «pensiero unico» troverebbe un forte, insormontabile ostacolo, proprio nella Chiesa cattolica, di cui Marino acutamente individua l'attuale, importante ruolo di contrappeso e ne intuisce la valenza di un impegno proiettato nel futuro. Papa Francesco non sottovaluta la perversa tendenza delle forze economiche a imporre la propria egemonia. Parla anche, per adeguarsi al linguaggio corrente e per farsi capire, di «pensiero unico». E mette in guardia i cattolici dal rischio che essi corrono di perdere la loro identità. Ma il suo non è un allarme disperato. Egli sa che le forze malefiche «non praevalebunt» e sollecita il ricorso agli efficaci deterrenti di cui i credenti dispongono per potere responsabilmente allontanare il pericolo. Bisogna agire, prima che i danni siano irreparabili per la nostra epoca. «Il fenomeno del pensiero unico», secondo il Pontefice, ha sempre causato "disgrazie nella storia dell'umanità», così come è avvenuto con le tragedie delle recenti dittature del novecento, tendenti a imporre il dominio l'una sull'altra («L'Osservatore Romano», 11 aprile $2014)^{9}$.

La ultra-millenaria storia della Chiesa, malgrado le molte gravi deviazioni e talune altrettanto gravi depravazioni, addebitabili alla debolezza dei suoi uomini e non alla sacralità dell'istituzione, è di tutta garanzia per l'umanità. La Chiesa ha anche esperienza nell'avere sconfitto la tendenza al «pensiero unico» di altre epoche storiche e di altri vasti movimenti ideologici. Basta ricordare il primo avvenimento della serie, allorquando, essa, affrontando il martirio, lievitò in senso cristiano la civiltà romana sino ad assimilarla. L'impero divenne cristiano e la Chiesa ne ereditò la struttura e l'universalismo.

In un clima di diffuso e condiviso ecumenismo anche le numerose confessioni di matrice cristiana, con tutta la loro persistente eterogeneità dottrinale, costituiscono, assieme alla Chiesa cattolica, un «fronte unico» contro la tendenza egemonica neocapitalista. Lo si

\footnotetext{
${ }^{9}$ Meditazione mattutina di Papa Francesco nella Cappella della Domus Sanctae Marthae, La dittatura del pensiero unico, pronunciata il 10 aprile 2014, in L'Osservatore Romano, CLIV, 83, 11 aprile 2014, URL: <https://w2.vatican.va/content/francesco/it/cotidie/2014/documents/papa-francescocotidie_20140410_dittatura-del-pensiero-unico.html> [consultato il 19 luglio 2018].
} 
intravede, in materia sociale e socio-economica, nell'unanime consenso di queste confessioni al magistero pontificio, tutte d'intesa a combattere la cosiddetta «società dei consumi» e il consumismo. Il neocapitalismo occidentale ha, certamente, contribuito al fallimento della Unione Sovietica, ma dall'esterno e in maniera parziale, perché è stata la profonda coscienza religiosa del popolo russo e dei popoli affiliati a sgretolare dall'interno le basi ideologiche e le strutture del colosso ateo e materialista.

A tal riguardo non bisogna dimenticare che è storicamente accertato che, in Polonia, il cardinale Karol Józef Wojtyla, papa san Giovanni Paolo II, e Lech Wałęsa con il sindacato «Solidarność» di matrice cristiana, non solo si resero interpreti di tutto un movimento nazionale e federale, ma furono anche, in gran parte, diretti ispiratori della rivoluzione anticomunista che ebbe il culmine con il crollo del Muro di Berlino ${ }^{10}$. La storia di Michail Gorbaciov e l'odierna politica di Vladimir Putin in materia religiosa stanno, fra l'altro, a dimostrare quale e quanto peso ha avuto e ha la stessa Chiesa ortodossa sulle vicende personali e sul ruolo pubblico dei potenti inquilini del Cremlino.

È esatto, a mio parere, ciò che scrive Marino, secondo il quale la Perestrojka di Michail Gorbaciov $^{11}$ "coniugava il tentativo di rinnovare il comunismo dell'Urss con l'ipotesi di un possibile recupero dialettico, da parte della realtà sovietica ormai in grave crisi, delle conquiste di libertà della democrazia liberale» ${ }^{12}$. Ma occorre aggiungere che in tale processo ebbe la sua parte la coscienza critica cristiana del popolo sovietico, pubblicamente rappresentata proprio da Giovanni Paolo II, che sentì di operare in nome del cristianesimo piuttosto che per solo conto del cattolicesimo, dando voce e coraggio anche alla religione ortodossa e alle altre confessioni cristiane dell'Europa dell'Est.

Le parole da lui pronunciate il 22 ottobre 1978, nell'omelia per l'inizio del pontificato, ebbero l'effetto di un terremoto nell'Unione delle Repubbliche Socialiste Sovietiche e nel resto del mondo comunista. Ad appena undici anni dopo sarebbe, appunto, accaduto l'inimmaginabile: il crollo del Muro di Berlino. Per la loro immediata efficacia politica e, oggi, per il loro valore storico, è opportuno ricordare quelle parole, nelle quali ancora riecheggia un contenuto intensamente profetico: «Non abbiate paura! Aprite, anzi, spalancate le porte a Cristo! Alla sua salvatrice potestà aprite i confini degli Stati, i sistemi economici come quelli politici, i vasti campi di cultura, di civiltà, di sviluppo. Non abbiate paura! $»^{13}$.

\footnotetext{
${ }^{10}$ Cfr. POMIAN, Krzysztof, Polonia: sfida all'impossibile? Dalla rivolta di Poznan a Solidarnosc, Venezia, Marsilio, 1983.

${ }^{11}$ Si veda ad esempio GORBACHEV, Mikhail, MLYNAR Zdenek, Conversations with Gorbachev: on Perestroika, the Prague spring, and the crossroads of socialism, New York, Columbia University Press, 2002; RUDE, Gerd, Enigma Gorbaciov, Milano, SugarCo, 1991; GALEOTTI, Mark, Gorbachev and his revolution, Basingstoke, London, Macmillan, New York, St. Martin's Press, 1997.

${ }^{12}$ MARINO, Giuseppe Carlo, Un'età contro la storia. Saggio sulla rivoluzione del XXI secolo, cit., p. 29.

13 Omelia di Giovanni Paolo II per l'inizio del Pontificato, pronunciata il 22 ottobre 1978, URL: <https://w2.vatican.va/content/john-paul-ii/it/homilies/1978/documents/hf_jp-ii_hom_19781022_inizio-
} 
È superfluo sottolineare e provare come e quanto queste parole abbiano segnato la storia del secolo XX. Mi limito soltanto a ricordarne due significative pagine: la visita pastorale di Wojtyla in Germania nel giugno del 1996 con il memorabile discorso pronunciato davanti alla Porta di Brandeburgo e l'altra visita pastorale del gennaio 1998 a Cuba, laddove a L'Avana, in piazza José Martí, primeggiava sopra il palco papale l'immagine di Cristo. E durante la celebrazione della messa, le telecamere dei giornalisti, provenienti da gran parte dei Paesi del pianeta, erano puntate sulla prima fila per riprendere Fidel Castro più volte colto mentre applaudiva il Pontefice romano. Si dirà che il tutto fosse occasionale e in omaggio all'eccezionale ospite, ma manifestazioni del genere in precedenza erano impensabili, proprio perché inconciliabili con l'ideologia e la politica del regime ${ }^{14}$.

Ci troviamo, ovviamente, dinanzi a segnali di capovolgimento del corso della storia, i quali agli occhi dei credenti convalidano, anche in questa nostra epoca, l'affermazione ricavabile dagli scritti di Agostino d'Ippona che la Chiesa è destinata a essere sempre combattuta, ma a non essere mai vinta. Alla luce di una concezione provvidenzialistica della storia, che, in senso laico, fu anche di Gian Battista Vico, l'odierno impatto tra l'Occidente cristiano e il mondo islamico dovrebbe, nel corrente secolo o nei successivi, risolversi a vantaggio del primo. Un evento del genere trova spazio nella teoria della storia ideale eterna del filosofo napoletano che ammette l'eterogenesi dei fini e, in pari tempo, insiste sulla libertà dell'uomo quale unico artefice delle sue singole azioni in un contesto universale tutelato dalla protezione divina ${ }^{15}$. La strategia terroristica adottata dalle frange estremiste islamiche non sarebbe altro che un fenomeno di debolezza. Il ricorso alla violenza sarebbe provocato, non solo dal delirio di potenza di alcuni capi religiosi e politici, ma anche dalla raggiunta loro consapevolezza dell'inefficacia della ragione e dei mezzi pacifici nell'attività di proselitismo.

Condivido, intanto, con uguale senso di amarezza, la constatazione dell'autore secondo cui «la storia, che per le travolte generazioni della modernità era nelle scuole la materia più accetta e valorizzata, è diventata quasi certamente la più ostica e la meno compresa ed apprezzata dagli allievi e quasi sopportata come un incomodo dagli stessi programmatori dell'educazione». È accaduto che "l'età della storia", "pervenuta a piena maturità nell'Ottocento e poi per qualche decennio preservatasi nel trascinamento novecentesco dei suoi processi culturali, con la rottura della modernità determinata dalla rivoluzione

pontificato.html> [consultato il 19 luglio 2018].

${ }^{14}$ Visita pastorale in Germania di Giovanni Paolo II (21-23 giugno 1996), Discorso alla Cerimonia di Congedo di Giovanni Paolo II, pronunciato alla Porta di Brandeburgo (Berlino) il 23 giugno 1996, URL: $<$ https://w2.vatican.va/content/john-paul-ii/it/speeches/1996/june/documents/hf_jp-

ii_spe_19960623_berlino.html> [consultato il 19 luglio 2018]; Viaggio apostolico di Sua Santità Giovanni Paolo II a Cuba (21-26 gennaio 1998), Omelia di Giovanni Paolo II, pronunciata il 25 gennaio 1998, link: <https://w2.vatican.va/content/john-paul-ii/it/homilies/1998/documents/hf_jpii_hom_19980125_lahabana.html> [consultato il 19 luglio 2018].

${ }_{15}^{15}$ VICO, Giambattista, Principes d'une science nouvelle relative a la nature commune des nations, Paris, Nagel, 1953; BADALONI, Nicola (a cura di), Giambattista Vico, Opere filosofiche, Firenze, Sansoni, 1971. 
elettronico-informatica (che dalla fine del Novecento sta continuando ai nostri giorni), si è drasticamente convertita in un'“età dell'antistoria"»". Altre crisi analoghe, se non proprio identiche, sono state vissute e superate dalla cultura occidentale. La più grave, anche perché provocata da radicali motivazioni di tipo razionalistico, fu quella insorta nel Settecento illuminista. Si trattò, in effetti, di una risoluta ostilità verso la tradizione: una valanga che colpì le religioni e, in particolare, la religione cattolica, la storia in generale e, specificatamente, il Medio Evo. Eppure la reazione romantica, nel secolo successivo, generò un nuovo germoglio storiografico e letterario senza precedenti.

È categoricamente da escludere che la presunta, rivoluzionaria, discutibile tendenza verso un «pensiero unico» possa di conseguenza determinare la «fine della storia». Anche se, per assurdo, il neocapitalismo giungesse a porre e a imporre l'egemonia sui cervelli umani, la storia non potrebbe finire. Avrebbe un nuovo corso, certamente diverso dall'attuale e indagabile con adeguate metodologie di ricerca, ma non si incamminerebbe nel viale del tramonto. Essa è il racconto della vita dell'uomo, delle vicende buone e cattive dell'umanità. E fintantoché l'uomo, libero artefice delle sue azioni, esisterà e sarà in grado, con «distensio animi», di dare consistenza e misura al tempo, la storia non potrà esaurirsi. Lo sostiene, in altre parole, Giuseppe Carlo Marino, che, privandoci di una pur attesa tirata delle somme della sua interessante riflessione, ne anticipa le conclusioni sin dalle prime pagine del saggio, laddove scrive che "per uno storico - quale che sia il livello delle sue ricerche sul passato e sul presente - soltanto la realtà dell'umano è storia e la storiografia è il suo proprio, "legittimo", percorso nel tempo storico di cui si costruisce il sapere, periodizzandone, affinandone, conservandone e tramandandone la memoria» ${ }^{17}$.

La storia non sarebbe finita neanche se si fosse avverata l'utopia marxiana della piena realizzazione del socialismo scientifico con l'avvento del comunismo e di una società senza classi. Il presunto, agognato, nuovo, immanente Eden, nonostante privo di un Dio, tuttavia sarebbe stato sempre abitato dall'uomo e, per giunta, da un uomo emancipato, assolutamente libero, «non alienato» né dal lavoro, né da una qualsivoglia forma religiosa. Quest'uomo, come nel passato, sarebbe stato ancora artefice della sua vita e, assieme ai suoi simili, di una nuova storia: magari senza classi e senza il motore etichettato «lotta di classe», ma una nuova storia, sempre nell'ambito delle categorie del tempo e dello spazio. Siffatta considerazione conclusiva, forse, è del tutto fuori luogo, perché nel mondo dell'utopia tutto è possibile. Anche l'impossibile.

\footnotetext{
${ }^{16}$ MARINO, Giuseppe Carlo, Un'età contro la storia. Saggio sulla rivoluzione del XXI secolo, cit., p. 52.

${ }^{17}$ Ibidem, p. 10.
} 


\section{L'AUTORE}

Eugenio GUCCIONE è professore di Storia delle dottrine politiche all'Università di Palermo. È stato per molti anni direttore dell'Istituto di Studi Storici della Facoltà di Scienze Politiche, della quale per più di un decennio è stato il decano. È direttore scientifico della nuova serie della rivista quadrimestrale «Storia e Politica», edita dal Dipartimento di Studi Europei dell'Università di Palermo. Collabora alla «Nuova Antologia». Dal 1954 fa parte del Movimento Federalista Europeo. Si è occupato del pensiero politico italiano e francese del XIX e XX secolo con ricerche sul cristianesimo sociale, sul cooperativismo, sul federalismo e sul rapporto tra la cultura laica e il movimento cattolico. Ha recuperato e curato, con ampia presentazione e note, un inedito di Gioacchino Ventura, venuto alla luce dopo più di un secolo e mezzo, dal significativo titolo Dello spirito della rivoluzione e dei mezzi di farla terminare.

URL: $<$ http://www.studistorici.com/progett/autori/\#Guccione $>$ 